\title{
Contemporary diagnostic IMAGing OF DISEASES OF THE SMALL INTESTINE - MAGNETIC RESONANCE ENTEROCLYSIS AND ENTEROGRAPHY
}

\author{
Małgorzata Dobrowolska-Bąk ${ }^{\mathrm{A}, \mathrm{B}, \mathrm{C}, \mathrm{D}}$, Anna Dubis ${ }^{\mathrm{A}, \mathrm{D}, \mathrm{E}, \mathrm{F}}$, Paulina Karcz ${ }^{\mathrm{B}}$, Karolina Rożnawska ${ }^{\mathrm{B}}$
}

Department of Electro-radiology, Institute of Physiotherapy, Faculty of Health Sciences, Jagiellonian University Medical College, Krakow, Poland

Authors' contribution:

A. Study design/planning • B. Data collection/entry $\bullet$ C. Data analysis/statistics $\bullet$ D. Data interpretation $\bullet$ E. Preparation of manuscript $\bullet$ F. Literature analysis/search $\bullet$ G. Funds collection

\author{
Address for correspondence: \\ Małgorzata Dobrowolska-Bąk \\ Department of Electro-radiology \\ Institute of Physiotherapy \\ Faculty of Health Sciences \\ Jagiellonian University Medical College \\ 12 Michałowskiego St. \\ 31-126 Krakow, Poland \\ e-mail: malgorzata.dobrowolska-bak@uj.edu.pl \\ SUBMITTED: 25.11.2019 \\ ACCEPTED: 10.12 .2019 \\ DOI: https://doi.org/10.5114/ppiel.2019.92533
}

\begin{abstract}
Examination of intestines using methods of magnetic resonance (MR) enterography and MR enteroclysis is currently considered to be the best way to visualise diseases of the small intestine like Crohn's disease, other inflammatory diseases of the small intestine, celiac disease, small intestine tumours, and cancers. They are both used in the initial diagnostics, assessment of the severity, as well as in monitoring of the course of the disease and possible complications. Both diagnostic methods require initial preparation of the patient by oral administration of a contrast agent. There are three types of oral contrast agents used in MR enterography and MR enteroclysis: positive, negative, and biphasic. In addition, an intravenous gadolinium contrast agent is administered during the study. MR enterography and MR enteroclysis allow accurate assessment of such structures as: the thickness of the intestinal wall, layering of the intestinal wall, narrowing, obstruction, gastrointestinal fistulas, gastrointestinal adipose tissue, encapsulated or free fluid in the abdomen, and lymph nodes. The undoubted advantages of these diagnostic methods include the lack of invasiveness, the possibility of obtaining multifaceted images, the possibility of assessing pathology not only within the intestine, but also in parenteral localisation, and the total absence of exposure of the patient to ionising radiation. The quality of the MR images obtained using both techniques depends largely on the correct preparation of the patient before the examination, in which the nurse participates to a large extent, leading the correct cooperation of the patient with the medical staff performing the MRI examination.
\end{abstract}

Key words: diagnostic imaging, magnetic resonance, small intestine, enterography, nurse's role.

\section{INTRODUCTION}

In recent years, significant progress has been observed in the imaging diagnostics of the small intestine. Classical imaging techniques such as gastrointestinal passage using barium sulphate have been largely replaced by magnetic resonance imaging (MRI) techniques [1, 2]. Intestinal MRI tests, due to their specificity, require special involvement of medical staff, especially nurses. The nurse's role is important due to the necessity of correct preparation of the patient prior to examination, preparation of the enteric contrast agent, monitoring appropriate hydration, overseeing possible complications associated with administration of the contrast agent (e.g. vomiting may occur in some patients), and aftercare following the examination due to the possibility of side effects (e.g. diarrhoea, hypotension).
Currently, there are two MRI techniques used in the imaging diagnostics of the small intestines: MR enterography and MR enteroclysis. Both methods are known as MRE, and both provide information on intestinal morphology (assessment of intestinal lumen and its wall) and surrounding tissues (intestinal fat, mesentery, lymph nodes). The main difference between these two imaging methods is the means of enteral administration of the contrast agent: in MR enteroclysis, the naso-intestinal probe is inserted into the duodenum near the Treitz ligament; while in MR enterography the agent is administered orally. Comparing both methods, it is worth noting that MR enteroclysis allows for a better and more accurate assessment of the intestinal lumen - especially the jejunum; therefore, it is helpful in diagnosing inflammatory diseases and cancers of the small intestine. It can be used as a first-line examination technique. 
Table 1. Indications for MR intestine imaging

\begin{tabular}{ll}
\hline \multicolumn{2}{l}{ Indications } \\
\hline 1. & Crohn's disease \\
\hline 2. & Other inflammatory diseases of the small intestine \\
\hline 3. & Celiac disease \\
\hline 4. & Small intestine tumours and cancers \\
\hline
\end{tabular}

MR enterography, on the other hand, is usually used to diagnose and monitor patients with Crohn's disease, diseases without jejunum involvement, and is a method of choice in paediatric patients in whom insertion of the naso-intestinal probe may be problematic [1, 3-7].

MRI technique allows for early detection of structural intestinal abnormalities and assessment of the severity of changes, which in turn accelerates the treatment process and hence improves patient care.

Additional advantages of MRI techniques are avoidance of ionising radiation and achievement of multi-faceted imaging with high resolution. One of the most common indications for MRI of the small intestine is assessment of the severity of changes seen in patients with Crohn's disease [4, 6-8]. Table 1 shows indications for this type of imaging.

\section{CONTRAST AGENTS USED IN INTESTINAL MRI}

Enteric contrast agents used in the imaging of the small intestine must meet certain criteria. Their desirable characteristics include, among others: even distribution, appropriate expansion of the lumen of the small intestine, and achievement of high contrast between the lumen and the intestinal wall. Contrast agents should not be absorbed and should not stimulate intestinal peristalsis. They should be well tolerated and easy to use.

There are three main groups of oral and enteric contrast agents: positive contrast agents, negative contrast agents, and two-phase contrast agents.

\section{Positive oral enteral contrast agents}

Positive contrast agents have paramagnetic properties. They allow high intensity of intestinal content signal in $\mathrm{T} 1$ and $\mathrm{T} 2$ dependent images to be obtained by reducing $\mathrm{T} 1$ relaxation time. They also allow imaging in the T1 sequence of the dependent thickening of the intestinal wall, but their disadvantage is the equalisation of the contrast between the intestinal lumen and the intestinal wall after intravenous administration. Positive contrast agents include: gadolinium chelates, manganese ions, ferrous ions, oily agents, e.g. corn oil, children's conditioner, and lowconcentration barium suspensions. The use of these agents is not routinely recommended [1, 5, 7].

\section{Negative oral enteral contrast agents}

Negative contrast agents give low signal intensity on T2- and T1-dependent images. These include solutions with superparamagnetic properties such as iron oxides (SPIO), diamagnetic contrast agents (high concentration barium sulphate), and agents with free protons, e.g. gases. Contrast agents containing superparamagnetic iron particles had been withdrawn from use due to side effects such as diarrhoea and nausea $[1,5,7]$.

\section{Biphasic oral enteral contrast agents}

Biphasic contrast agents give high or low signal intensity depending on the type of sequence used in the imaging process. They reduce the intensity of the lumen signal filled with the agent in the T1-dependent sequence but increase in the T2-dependent sequence. The low signal intensity of these agents in T1-dependent sequences allows for improved contrast between intestinal lumen and, for example, an inflammatory wall after intravenous administration of the contrast agent. Similarly, the T2-dependent sequence without intravenous administration of the contrast agent results in better contrast imaging between high signal of intestinal lumen and low signal of intestinal wall.

These include substances containing manganese (blackcurrant juice, green tea), water, ferric ammonium citrate, methyl cellulose, mannitol, polyethylene glycol, and sorbitol. Due to possible side effects such as nausea, vomiting, and diarrhoea, in some diagnostic centres metoclopramide is used to minimise them $[1,2,5,7]$.

\section{THE NURSE'S ROLE IN PATIENT PREPARATION FOR THE MRI EXAMINATION}

Achievement of proper and good quality diagnostic images in MR enteroclysis and enterography requires appropriate preparation of a patient for the examination (hospital ward) or provision of correct information regarding home preparation prior to imaging appointment. An important role here is played by the nurse (MRI laboratory or hospital ward). The nurse provides all necessary information about the preparation and the entire examination to the patient. In addition, the nurse overlooks the proper course of the preparation for the study in the MRI laboratory.

Approximately 2-3 days before the examination, the patient should eat a light digestible diet, sometimes even a semi-liquid one, eliminating from the diet dairy products, meals rich in carbohydrates, as well as large quantities of food. The day before the examination (if the imaging is scheduled for the morning the next day) or on the day of the exami- 
nation (if the examination is scheduled for the afternoon), the patient should perform a rectal infusion using a ready-made enema kit. This is not necessary in most cases in patients who have diarrhoea. Before the intestinal MR examination, the patient should be fasting - without food for about 4-6 hours. Moreover, an intravenous contrast agent is used during the examination. Therefore, the patient must obtain renal function test results (creatinine level and eGFR) prior to date of the test [3].

The patient should report to the MRI laboratory about an hour before the scheduled date of the examination - the second part of patient preparation takes place in the laboratory under the supervision of a nurse. His or her tasks include preparation of the patient's intravenous puncture (in the case of absence of any contraindications, the intravenous contrast agent is administered during the study) and preparation of the oral contrast agent, which the patient receives to drink (50-60 min before the scheduled time of the study) $[3,4]$. The time of administration of the oral contrast agent should be recorded in the patient's file. In the case of MR enterography, an appropriate proportion of mannitol or sorbitol is diluted with water - the patient receives one litre of ready-made preparation to drink. In order to minimise motor artefacts associated with physiological intestinal peristalsis, antispasmodics such as hyoscine butylbromide (Buscopan $\left.{ }^{\circledR}\right)$ or drotaverine hydrochloride (No-spa $\left.{ }^{\circledR}\right)$ are used during the study [1]. If Buscopan ${ }^{\circledR}$ is given, it is necessary to collect a history of glaucoma because its use is contraindicated in patients with this condition.

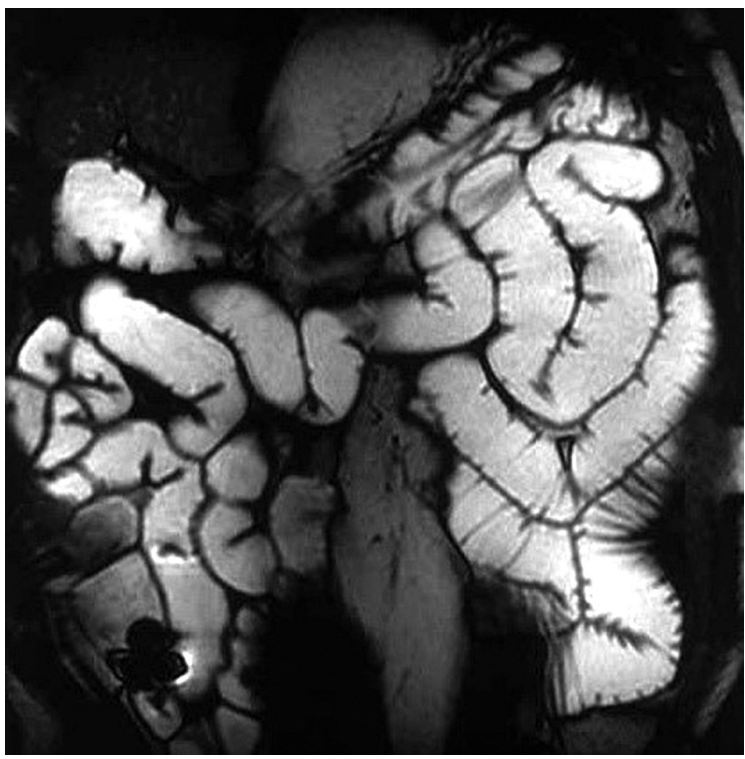

Figure 1. MR enterography study. Correct filling of the intestines with an oral contrast agent (source: MR MCD Voxel Laboratory archive)
Correct patient preparation is assessed on the basis of the first sequences of the MRI examination. A properly prepared small intestine should be about $2.5-3 \mathrm{~cm}$ wide. This is important because of the risk of false-negative results (oversight) or false-positive results (a collapsed intestine may suggest thickening of its wall). Figures 1 and 2 show correct and abnormal bowel filling with an orally administered contrast agent, respectively.

\section{PREPARATION OF THE PATIENT FOR MR ENTEROCLYSIS}

MR enteroclysis is a study in which the contrast agent is administered through an inserted naso-intestinal probe, the end of which should be placed in the duodenum near the Treitz ligament [1, 4-7]. The correct position of the probe should be confirmed by radiological examination - fluoroscopy, using ionising radiation. Therefore, it is definitely a rarely chosen method for imaging of the small intestine in paediatrics.

The contrast medium is administered by means of a pump compatible with the MR apparatus at a rate of about $80-200 \mathrm{ml} / \mathrm{min}$.

\section{METHOD OF CONDUCTING THE MR EXAMINATION}

A patient properly prepared by the nurse for the examination (metal elements of clothing and jewellery removed) is placed in the MRI scanner in a prone position, which further minimises intestinal peristal-

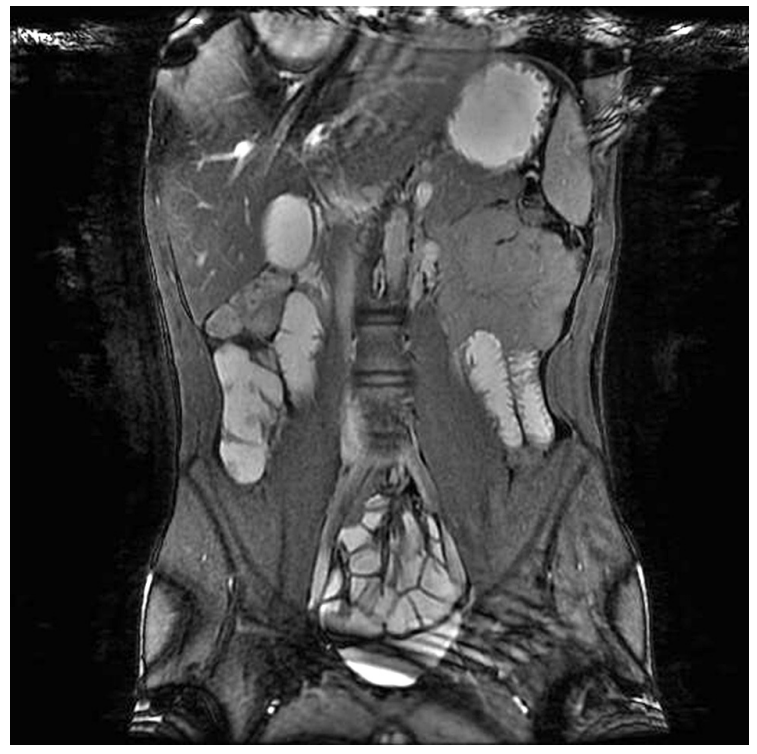

Figure 2. MR enterography study. Incorrect filling of the intestines with an oral contrast agent (source: MR MCD Voxel Laboratory archive) 
Table 2. Structures analysed in the bowel MRI examinations

\begin{tabular}{lll}
\hline No. & Analysed structure & Analysis \\
\hline 1. & The thickness of the intestinal wall & $\begin{array}{l}\text { With correctly inflated walls should be about 1-3 mm. Thickening } \\
\text { of the wall above } 3 \mathrm{~mm} \text { indicates a pathological process, e.g. in } \\
\text { Crohn's disease, ischaemic diseases, or graft versus host disease }\end{array}$ \\
\hline 2. & Layering of the intestinal wall & Crohn's disease staging \\
\hline 3. & Narrowing, obstruction & Determination of the severity \\
\hline 4. & $\begin{array}{l}\text { Gastrointestinal fistulas, gastrointestinal adipose } \\
\text { tissue, its degree of infiltration, presence of abscesses }\end{array}$ & Determination of the severity \\
\hline 5. & Encapsulated or free fluid in the abdomen & Determination of the severity \\
\hline 6. & Lymph nodes & Determination of the severity \\
\hline
\end{tabular}

tic movements. This position also allows for additional widening of the intestinal lumen.

The patient receives from the nurse earphones for noise suppression during the examination. The test takes about 40 minutes to complete. The key to a successful examination is patient cooperation following instructions received from the examining technician. The correct way to hold one's breath and remain still during the examination are parts of those instructions, both of which aim to reduce the formation of motion and respiratory artefacts, thus allowing obtaining good quality diagnostic images. During all exams the nurse supervises the patient's wellness via a glass window and microphone connection.

Various MR sequences are used during MRI testing, including dynamic (cine) sequences used to assess intestinal motility.

In addition to oral/enteral contrasting agents, standard gadolinium preparations are administered by the nurse intravenously. They support diagnostic possibilities in both MRI methods, MR enteroclyses and MR enterographies, aiding the process of assessment of the normal gastrointestinal mucosa and assessment of pathologically changed intestinal walls (e.g. inflammatory, cancerous infiltration) [5-8]. Table 2 shows the structures that are analysed during bowel MRI.

\section{PATIENT CARE AFTER EXAMINATION}

After the examination, the patient remains with the intravenous puncture under the care of a nurse for about 20-30 minutes. This is due to the possibility of side effects in patients associated with both oral and intravenous contrast media administration.

Complications after administration of oral contrast agents include diarrhoea (caused by the rapid displacement of water into the lumen of the gastrointestinal tract), as well as blood pressure drop. If this occurs, a patient requires longer observation, blood pressure monitoring, and intravenous administration of electrolyte-containing fluids.

\section{Disclosure}

The authors declare no conflict of interest.

\section{References}

1. Masselli G, Gualdi G. MR imaging of the small bowel. Radiology 2012; 264: 333-348.

2. Sinha R. Recent advances in intestinal imaging. Indian J Radiol Imaging 2011; 21: 170-175.

3. Mollard BJ, Smith EA, Dillman JR. Pediatric MR enterography: technique and approach to interpretation-how we do it. Radiology 2015; 274: 29-43.

4. Griffin N, Grant LA, Anderson S, et al. Small bowel MR enterography: problem solving in Crohn's disease. Insights Imaging 2012; 3: 251-263.

5. Gee MS, Harisinghani MG. MRI in patients with inflammatory bowel disease. J Magn Reson Imaging 2011; 33: 527-534.

6. Amzallag-Bellenger E, Oudjit A, Ruiz A, et al. Effectiveness of MR enterography for the assessment of small-bowel diseases beyond Crohn disease. Radiographics 2012; 32: 1423-1444.

7. Tolan DJM, Greenhalgh R, Zealley IA, et al. MR Enterographic Manifestations of Small Bowel Crohn Disease. RadioGraphics 2010; 30: 367-384.

8. Martin DR, Lauenstein T, Sitaraman SV. Utility of magnetic resonance imaging in small bowel crohn's disease. Gastroenterology 2007; 133: 385-390. 